\title{
Spherical Lamina Emergent Mechanisms
}

Samuel E. Wilding

Brigham Young University - Provo

Larry L. Howell

Brigham Young University - Utah, Ihowell@byu.edu

Spencer P. Magleby

Follow this and additional works at: https://scholarsarchive.byu.edu/facpub

Part of the Mechanical Engineering Commons

\section{Original Publication Citation}

Wilding, S.E., Howell, L.L., Magleby, S.P., “Introduction of Planar Compliant Joints Designed for Compressive and Tensile Loading Conditions in Lamina Emergent Mechanisms," Mechanism and Machine Theory, Vol. 56, pp. 1-15, DOI: 10.1016/j.mechmachtheory.2012.05.007, 2012.

\section{BYU ScholarsArchive Citation}

Wilding, Samuel E.; Howell, Larry L.; and Magleby, Spencer P., "Spherical Lamina Emergent Mechanisms" (2012). Faculty Publications. 1556.

https://scholarsarchive.byu.edu/facpub/1556

This Peer-Reviewed Article is brought to you for free and open access by BYU ScholarsArchive. It has been accepted for inclusion in Faculty Publications by an authorized administrator of BYU ScholarsArchive. For more information, please contact ellen_amatangelo@byu.edu. 


\title{
Spherical Lamina Emergent Mechanisms
}

\author{
Samuel E. Wilding, Larry L. Howell*, Spencer P. Magleby \\ Brigham Young University, Department of Mechanical Engineering, Provo, UT 84602, United States
}

\begin{abstract}
Lamina emergent mechanisms (LEMs) are compliant mechanisms that can be manufactured from planar materials and are capable of motion out of the plane of manufacture. Spherical LEMs combine the unique motion of spherical mechanisms with the compactness and simple topology of LEMs. The fundamentals of spherical LEMs are discussed in this work. It is found that 21 of the 33 possible spherical $4 \mathrm{R}$ types can be spherical LEMs. A classification based on these 21 possible types is developed. This classification is used to predict motion capabilities of spherical 6R, and Equintet mechanisms. It is also applied to arrays of spherical mechanisms. The extreme compactness and sophisticated motion of spherical LEMs makes them candidates for application that are weight and volume sensitive and may find use in future surface morphing applications.
\end{abstract}

Keywords: Spherical Mechanisms, Compliant Mechanisms, Lamina Emergent Mechanisms, Surface Morphing

\section{Introduction}

The purpose of this paper is to introduce spherical lamina emergent mechanisms and to describe the fundamentals that govern them. Lamina emergent mechanisms (LEMs) are compliant mechanical devices that are fabricated from planar materials with motion out of the fabrication plane [1]. As compliant mechanisms [2], LEMs achieve their motion by the deflection of flexible members. LEMs feature simple topology, but are capable of performing sophisticated tasks. The planar nature of LEMs allows them to be highly compact and suitable for fabrication by simple manufacturing processes. Most of the developments and applications of LEMs have been dedicated to planar mechanisms. In this work, the capabilities and some of the possible applications of spherical LEMs are discussed.

For spherical mechanisms, any moving point is constrained to the surface of a sphere and the spherical surfaces are concentric [3]. Spherical mechanisms are the simplest mechanisms capable of providing spatial motion [4]. By their nature, spherical mechanisms are compact [3].

There is an opportunity to create mechanisms capable of spatial motion that are exceptionally compact by combining the spatial efficiency of spherical mechanisms and the planar nature of lamina emergent mechanisms. The combination is referenced to as "spherical lamina emergent mechanisms" and they show promise for applications where sophisticated motion is required in a small volume. This paper identifies and classifies possible spherical LEMs. The classification includes all possible spherical 4R LEMs. By decomposition, more advanced mechanisms can be classified. The classification aids in predicting mechanism motion and in determining which mechanisms might be appropriate for particular applications. The classification builds on the classification scheme developed by Cervantes-Sánchez et al. [5] for rigid-link spherical mechanisms. This classification represents all possible spherical 4R linkages. It also lists all possible mechanisms, based on limits of link motion. It is based solely on link lengths and thus is readily applicable to classification of spherical LEMs. Classification schemes group mechanisms based on there general characteristics These characteristics can be used as a first step of synthesis purposes [6] and provide an overview of the design space for the mechanisms they concern. Many have been developed for planar $[6,7,8,9]$, spherical $[3,5,9,10]$ and spatial mechanisms $[11]$.

\footnotetext{
${ }^{*}$ Corresponding Author. Tel.: +1 8014228037.

Email address: 1howell@byu. edu (Larry L. Howell)
} 


\section{Background}

Cervantes-Sánchez et al. [5] defined a classification scheme for spherical $4 \mathrm{R}$ linkages. They report there are 33 unique types of spherical $4 \mathrm{R}$ linkages. The classification is based on the range of motion of the links based on link lengths. Graphical representations of link mobility have also been developed by the same authors [12]. The mechanism types are distinguished by using designators of the form $(a, b, c, d)$ where $a, b, c$, and $d$ can take integer values from one to four and represent the limits of the angles $\theta, \beta, \gamma$, and $\phi$ (See Figure 1$)^{1}$. The values of $a, b, c$, and $d$ are determined using the following equations [5]:

Equations for $a$

$$
\begin{aligned}
& \cos \left(\alpha_{4}-\alpha_{3}\right) \geq \cos \left(\alpha_{1}-\alpha_{2}\right) \\
& \cos \left(\alpha_{3}+\alpha_{4}\right) \leq \cos \left(\alpha_{2}+\alpha_{1}\right)
\end{aligned}
$$

Equations for $b$

$$
\begin{aligned}
& \cos \left(\alpha_{1}-\alpha_{4}\right) \geq \cos \left(\alpha_{3}-\alpha_{2}\right) \\
& \cos \left(\alpha_{4}+\alpha_{1}\right) \leq \cos \left(\alpha_{2}+\alpha_{3}\right)
\end{aligned}
$$

Equations for $c$

$$
\begin{aligned}
& \cos \left(\alpha_{1}-\alpha_{2}\right) \geq \cos \left(\alpha_{4}-\alpha_{3}\right) \\
& \cos \left(\alpha_{2}+\alpha_{1}\right) \leq \cos \left(\alpha_{3}+\alpha_{4}\right)
\end{aligned}
$$

Equations for $d$

$$
\begin{aligned}
& \cos \left(\alpha_{3}-\alpha_{2}\right) \geq \cos \left(\alpha_{1}-\alpha_{4}\right) \\
& \cos \left(\alpha_{2}+\alpha_{3}\right) \leq \cos \left(\alpha_{4}+\alpha_{1}\right)
\end{aligned}
$$

The values of each of the designators based on Equations (1) through (8), as well as definition of each designator value, are shown in Table 1. The maximum and minimum values of each link angle are defined as

$$
\begin{aligned}
& \cos \left(\theta_{\text {max }}\right)=\frac{\cos \left(\alpha_{3}+\alpha_{4}\right)-\cos \left(\alpha_{2}\right) \cos \left(\alpha_{1}\right)}{\sin \left(\alpha_{2}\right) \sin \left(\alpha_{1}\right)} \\
& \cos \left(\theta_{\text {min }}\right)=\frac{\cos \left(\alpha_{4}-\alpha_{3}\right)-\cos \left(\alpha_{2}\right) \cos \left(\alpha_{1}\right)}{\sin \left(\alpha_{2}\right) \sin \left(\alpha_{1}\right)} \\
& \cos \left(\beta_{\text {max }}\right)=\frac{\cos \left(\alpha_{4}+\alpha_{1}\right)-\cos \left(\alpha_{2}\right) \cos \left(\alpha_{3}\right)}{\sin \left(\alpha_{2}\right) \sin \left(\alpha_{3}\right)} \\
& \cos \left(\beta_{\text {min }}\right)=\frac{\cos \left(\alpha_{1}-\alpha_{4}\right)-\cos \left(\alpha_{2}\right) \cos \left(\alpha_{3}\right)}{\sin \left(\alpha_{2}\right) \sin \left(\alpha_{3}\right)} \\
& \cos \left(\gamma_{\text {max }}\right)=\frac{\cos \left(\alpha_{2}+\alpha_{1}\right)-\cos \left(\alpha_{3}\right) \cos \left(\alpha_{4}\right)}{\sin \left(\alpha_{3}\right) \sin \left(\alpha_{4}\right)} \\
& \cos \left(\gamma_{\text {min }}\right)=\frac{\cos \left(\alpha_{1}-\alpha_{2}\right)-\cos \left(\alpha_{3}\right) \cos \left(\alpha_{4}\right)}{\sin \left(\alpha_{3}\right) \sin \left(\alpha_{4}\right)} \\
& \cos \left(\phi_{\text {max }}\right)=\frac{\cos \left(\alpha_{2}+\alpha_{3}\right)-\cos \left(\alpha_{4}\right) \cos \left(\alpha_{1}\right)}{\sin \left(\alpha_{4}\right) \sin \left(\alpha_{1}\right)} \\
& \cos \left(\phi_{\text {min }}\right)=\frac{\cos \left(\alpha_{3}-\alpha_{2}\right)-\cos \left(\alpha_{4}\right) \cos \left(\alpha_{1}\right)}{\sin \left(\alpha_{4}\right) \sin \left(\alpha_{1}\right)}
\end{aligned}
$$

\footnotetext{
${ }^{1}$ Note: Cervantes-Sánchez et al. [5] use different link subscripts, with $\alpha_{1}$ as the input link. In this work $\alpha_{1}$ is the ground link.
} 


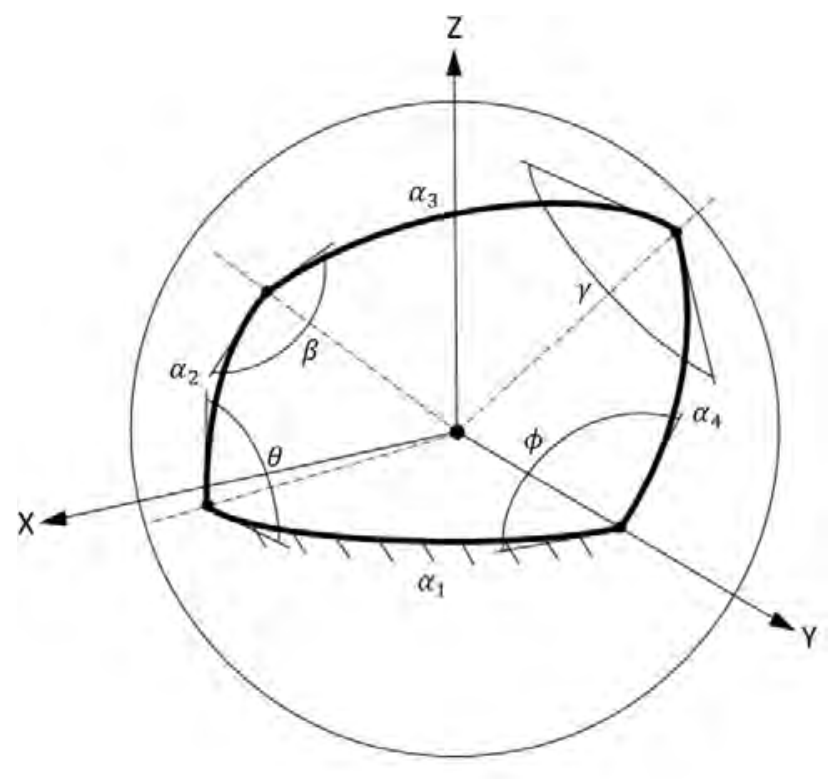

Figure 1: Skeleton Diagram of a Spherical 4R Mechanism

\section{Spherical LEMs}

Spherical LEMs combine the compactness and simple topology of LEMs with the compact nature and three dimensional motions of spherical mechanisms. Spherical LEMs have similar features to those described by Lusk et al. [13] for microscale spherical mechanisms, in that the mechanism is manufactured in a planar position, the mechanism base (the sheet the mechanism was cut from) can cause interference, and their are geometric limitations on the orientation of the joint axes. Some examples of spherical LEMs are shown in [1, 14, 15]. Many of the prototypes created for this work use creases as joints, but other joints like the Lamina Emergent Torsional (LET) [16] joint can also be used.

An example of a spherical 4R LEM is shown in Figure 2. This LEM was constructed of sheet steel. The joints have been plastically deformed, so that the mechanisms stays in a displaced position. The joints of this mechanism are LET joints which allow movement of the mechanism by torsion of long, thin segments [16]. This mechanism illustrates the planar nature of LEMs as well as the complex motion possible with spherical mechanisms.

For a spherical $4 \mathrm{R}$ mechanism to have a planar state (also referred to as the ability for a mechanism to fold [9]), one of the following equations must be true (see Figure 1 for link designations):

$$
\begin{aligned}
& \alpha_{1}+\alpha_{4}=\alpha_{2}+\alpha_{3} \\
& \alpha_{1}+\alpha_{2}=\alpha_{3}+\alpha_{4} \\
& \alpha_{1}+\alpha_{3}=\alpha_{2}+\alpha_{4}
\end{aligned}
$$

As will be shown, many spherical mechanisms and arrays of spherical mechanisms can be reduced to a series of $4 \mathrm{R}$ linkages, so the criteria established here can be applied to mechanisms beyond the $4 \mathrm{R}$ linkage.

A mechanism whose link lengths satisfy Equation (17), (18) or (19) will be said to belong to Spherical LEM Class Ia, Ib, or II, respectively. The Spherical LEM classes organize the possible spherical 4R LEMs based on their layout in the planar position. The classes ensure that the correct link length and angle notations are assigned. This is essential to ensuring accurate mechanism analysis. Table 2 shows the angles of each link in the mechanisms planar position (see Figure 1 for angle definitions). 
Table 1: Spherical Mechanism Designation Definitions [5]

\begin{tabular}{|c|c|c|c|}
\hline Equation 1 & Equation 2 & Value & Definition \\
\hline True & True & $a=1$ & $\theta$ has no upper or lower limit. The input link can fully rotate relative to the ground link. \\
\hline True & False & $a=2$ & $\theta$ has an upper limit. The input link rocks between $\pm \theta_{\max }$, passing through $\theta=0^{\circ}$. \\
\hline False & True & $a=3$ & $\theta$ has a lower limit. The input link rocks between $\pm \theta_{\min }$, passing through $\theta=180^{\circ}$. \\
\hline False & False & $a=4$ & $\theta$ has an upper and lower limit. The input link rocks between $\theta_{\min }$ and $\theta_{\max }$ or $-\theta_{\min }$ and $-\theta_{\max }$. \\
\hline Equation 3 & Equation 4 & Value & Definition \\
\hline True & True & $b=1$ & $\beta$ has no upper or lower limit. The coupler can fully rotate relative to the input link. \\
\hline True & False & $b=2$ & $\beta$ has an upper limit. The coupler link rocks between $\pm \beta_{\max }$, passing through $\beta=0^{\circ}$. \\
\hline False & True & $b=3$ & $\beta$ has a lower limit. The coupler link rocks between $\pm \beta_{\min }$, passing through $\beta=180^{\circ}$. \\
\hline False & False & $b=4$ & $\beta$ has an upper and lower limit. The coupler link rocks between $\theta_{\min }$ and $\theta_{\max }$ or $-\theta_{\min }$ and $-\theta_{\max }$. \\
\hline Equation 5 & Equation 6 & Value & Definition \\
\hline True & True & $c=1$ & $\gamma$ has no upper or lower limit. The coupler can fully rotate relative to the output link. \\
\hline True & False & $c=2$ & $\gamma$ has an upper limit. The coupler link rocks between $\pm \gamma_{\max }$, passing through $\gamma=0^{\circ}$. \\
\hline False & True & $c=3$ & $\gamma$ has a lower limit. The coupler link rocks between $\pm \gamma_{\min }$, passing through $\gamma=180^{\circ}$. \\
\hline False & False & $c=4$ & $\gamma$ has an upper and lower limit. The coupler link rocks between $\gamma_{\min }$ and $\gamma_{\max }$ or $-\gamma_{\min }$ and $-\gamma_{\max }$. \\
\hline Equation 7 & Equation 8 & Value & Definition \\
\hline True & True & $d=1$ & $\phi$ has no upper or lower limit. The output link can fully rotate relative to the ground link. \\
\hline True & False & $d=2$ & $\phi$ has an upper limit. The input link rocks between $\pm \phi_{\max }$, passing through $\phi=0^{\circ}$. \\
\hline False & True & $d=3$ & $\phi$ has a lower limit. The input link rocks between $\pm \phi_{\min }$, passing through $\phi=180^{\circ}$. \\
\hline False & False & $d=4$ & $\phi$ has an upper and lower limit. The input link rocks between $\phi_{\min }$ and $\phi_{\max }$ or $-\phi_{\min }$ and $-\phi_{\max }$. \\
\hline
\end{tabular}

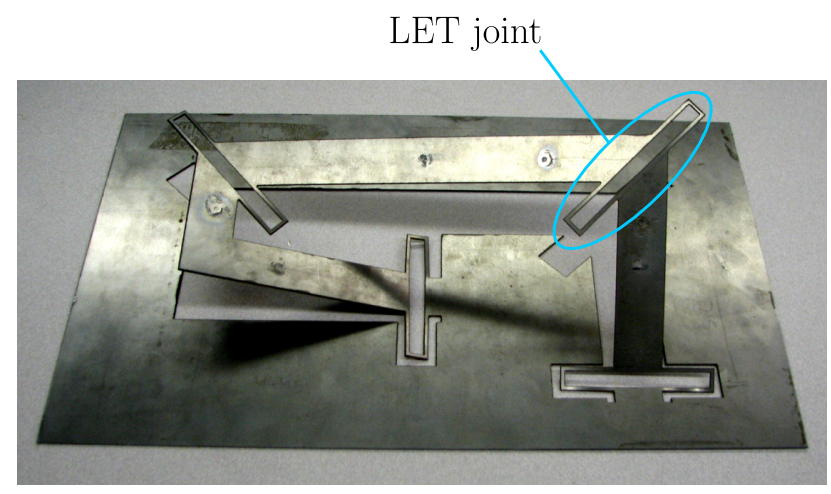

Figure 2: Spherical 4R LEM

Figure 3 shows an example of a spherical 4R LEM that would belong to each class. The lines with long dashes represent revolute joints, or compliant joints with equivalent motion. The coordinate system is the same as that shown in Figure 1. It should be noted that if the output link and input link are switched, a Class Ia mechanism becomes a Class Ib mechanism, and vica versa. The input link must be specified to determine if the mechanism belongs to Class Ia or Ib. Class II is independent of the other two classes in that switching the input and output of a Class II mechanism becomes a different mechanism type, but remains Class II. This is due to fact that Class II mechanisms lay in a "crossed" configuration in their planar state, meaning that the input and output links lay in opposite directions and will actuate in opposite directions, whereas for Class Ia and Class Ib, the input and output links lay in the same direction and actuate in the same direction. 
Table 2: Characteristic of Spherical LEM classes

\begin{tabular}{|c|c|c|c|}
\hline Class & \multicolumn{3}{|c|}{$\begin{array}{l}\text { Link Angle Conditions } \\
\text { in Planar Position }\end{array}$} \\
\hline \multirow{4}{*}{ Ia } & $\theta$ & $=$ & $0^{o}$ \\
\hline & $\beta$ & $=$ & $180^{\circ}$ \\
\hline & $\gamma$ & $=$ & $0^{\circ}$ \\
\hline & $\phi$ & $=$ & $180^{\circ}$ \\
\hline \multirow{4}{*}{$\mathrm{Ib}$} & $\theta$ & $=$ & $180^{\circ}$ \\
\hline & $\beta$ & $=$ & $0^{\circ}$ \\
\hline & $\gamma$ & $=$ & $180^{\circ}$ \\
\hline & $\phi$ & $=$ & $0^{\circ}$ \\
\hline \multirow{4}{*}{ II } & $\theta$ & $=$ & $0^{\circ}$ \\
\hline & $\beta$ & $=$ & $0^{\circ}$ \\
\hline & $\gamma$ & $=$ & $0^{o}$ \\
\hline & $\phi$ & $=$ & $0^{\circ}$ \\
\hline
\end{tabular}

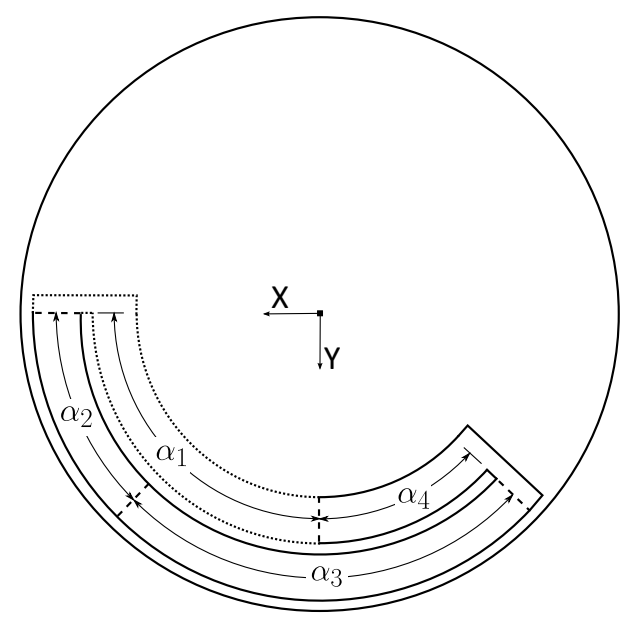

(a) Class Ia

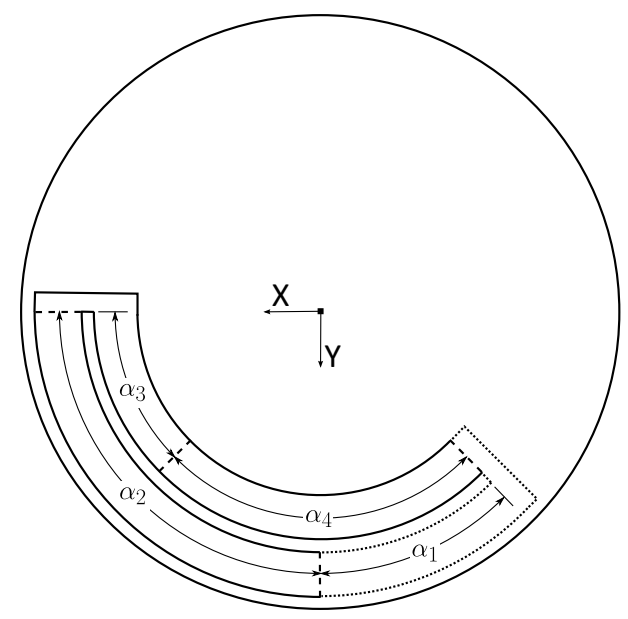

(b) Class Ib

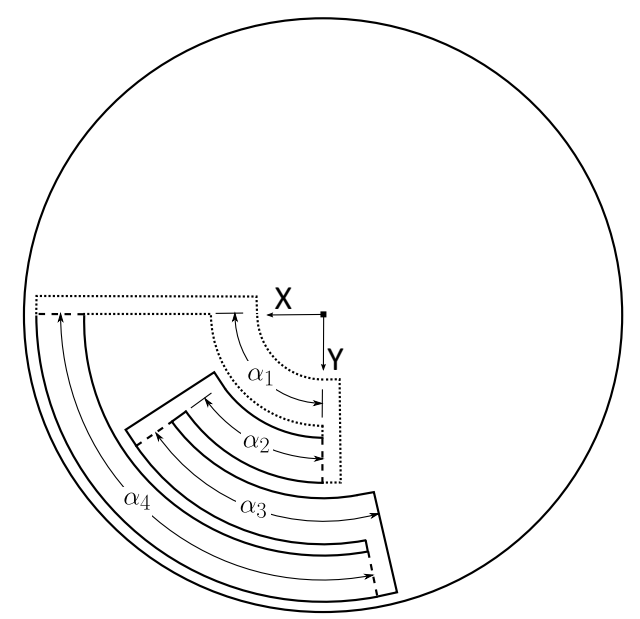

(c) Class II

Figure 3: Examples of mechanisms from the 3 classes 


\subsection{Class Ia}

To determine which mechanism types reported in [5] could be Class Ia spherical LEMs, Equation (17) was used in conjunction with Equations (1) through (8). An example of a Class Ia mechanism is shown in Figure 3(a). Combining Equations (17) and (1), we see that $\theta$ has no lower limit, thus $a \neq 3$ or 4 . By combining Equations (17) and (4), it is found that $\beta$ has no upper limit, thus $b \neq 2$ or 4. By similar methods, using Equations (5) and (8), $\gamma$ has no lower limit, $c \neq 3$ or $4, \phi$ has no lower limit, and $d \neq 2$ or 4 . The resulting possible mechanism types for Class Ia are listed in Table 3 .

\subsection{Class Ib}

An example of a Class Ib mechanism is shown in Figure 3(b). Using the same method as was used for Class Ia, Equation (18) was combined with Equations (2), (3), (6) and (7). Thus, there is no upper limit on $\theta$ or $\gamma$ and no upper limit on $\beta$ or $\phi$. Thus for a Class Ib, $a \neq 2$ or $4, b \neq 3$ or $4, c \neq 2$ or 4 , and $d \neq 3$ or 4 . The possible mechanism types for Class Ib are summarized in Table 3.

\subsection{Class II}

An example of a Class II mechanism is shown in Figure 3(c). Equation (19) was combined with Equations (1), (3), (5) and (7). Thus, there is no lower limit on $\theta, \beta, \gamma$, or $\phi$. For Class II, $a \neq 3$ or $4, b \neq 3$ or $4, c \neq 3$ or 4 , and $d \neq 3$ or 4 . The possible mechanism types for Class II are summarized in Table 3.

\begin{tabular}{|c|c|c|}
\hline Spherical LEM class & Type Numbers & Designation \\
\hline \multirow{9}{*}{ Class Ia } & 1 & $(2,3,1,1)$ \\
\hline & 2 & $(2,1,1,3)$ \\
\hline & 3 & $(1,3,2,1)$ \\
\hline & 4 & $(1,1,2,3)$ \\
\hline & 5 & $(2,1,1,1)$ \\
\hline & 6 & $(1,1,2,1)$ \\
\hline & 7 & $(1,3,1,1)$ \\
\hline & 8 & $(1,1,1,3)$ \\
\hline & 9 & $(1,1,1,1)$ \\
\hline \multirow{9}{*}{ Class Ib } & 1 & $(3,2,1,1)$ \\
\hline & 2 & $(3,1,1,2)$ \\
\hline & 3 & $(1,2,3,1)$ \\
\hline & 4 & $(1,1,3,2)$ \\
\hline & 5 & $(1,2,1,1)$ \\
\hline & 6 & $(1,1,1,2)$ \\
\hline & 7 & $(3,1,1,1)$ \\
\hline & 8 & $(1,1,3,1)$ \\
\hline & 9 & $(1,1,1,1)$ \\
\hline \multirow{9}{*}{ Class II } & 1 & $(2,2,1,1)$ \\
\hline & 2 & $(2,1,1,2)$ \\
\hline & 3 & $(1,2,2,1)$ \\
\hline & 4 & $(1,1,2,2)$ \\
\hline & 5 & $(2,1,1,1)$ \\
\hline & 6 & $(1,1,2,1)$ \\
\hline & 7 & $(1,2,1,1)$ \\
\hline & 8 & $(1,1,1,2)$ \\
\hline & 9 & $(1,1,1,1)$ \\
\hline
\end{tabular}

\subsection{Class Summary}

As shown in Table 3, there are 9 types of mechanism in each class, resulting in 27 mechanisms types. However, some mechanism types belong to multiple classes. If mechanism types that appear in multiple classes are only counted as one, it results in the 21 types (of the 33 mechanisms from [5]) that can be spherical LEMs. The situations that cause mechanism types to appear in multiple classes are listed in Table 4. In these cases, the orientation of the links must 
be known to determine the mechanism class because the link lengths satisfy more than one planar position condition (See Equations (17)-(18)). Additionally, eliminating isomorphisms (see [2]) results in only 15 unique spherical 4R LEM types. It is interesting to note that when a mechanism's link lengths satisfy the conditions to be a Class Ia or a Class Ib mechanism $\left(\alpha_{1}=\alpha_{3}\right.$ and $\left.\alpha_{2}=\alpha_{4}\right)$, the mechanism has two planar positions; one when $\theta=0^{\circ}$ and one when $\theta=180^{\circ}$ (see Figure 4). In this work, when the link lengths satisfy this condition the mechanism will be assumed to belong to Class Ia, meaning that the input link is chosen such that $\theta=0$ in the mechanisms first planar position.

Table 4: Similarity of the Mechanisms

\begin{tabular}{ll}
\hline Similar Mechanisms & Link Length Conditions \\
\hline $\begin{array}{l}\text { Class Ia Type } 9 \\
\text { Class Ib Type } 9\end{array}$ & $\alpha_{1}=\alpha_{3}$ and $\alpha_{2}=\alpha_{4}$ \\
\hline $\begin{array}{l}\text { Class Ia Type } 5 \\
\text { Class II Type } 5\end{array}$ & $\alpha_{1}=\alpha_{2}$ and $\alpha_{3}=\alpha_{4}$ \\
\hline $\begin{array}{l}\text { Class Ia Type } 6 \\
\text { Class II Type } 6\end{array}$ & $\alpha_{1}=\alpha_{2}$ and $\alpha_{3}=\alpha_{4}$ \\
\hline $\begin{array}{l}\text { Class Ib Type } 5 \\
\text { Class II Type } 7\end{array}$ & $\alpha_{1}=\alpha_{4}$ and $\alpha_{2}=\alpha_{3}$ \\
\hline $\begin{array}{l}\text { Class Ib Type } 6 \\
\text { Class II Type } 8\end{array}$ & $\alpha_{1}=\alpha_{4}$ and $\alpha_{2}=\alpha_{3}$ \\
\hline Class II Type 9 & $\alpha_{1}=\alpha_{2}=\alpha_{3}=\alpha_{4}$ \\
\hline
\end{tabular}

Table 5: The 15 unique Spherical 4R LEMs

\begin{tabular}{|c|c|}
\hline Number & Isomorphic Mechanisms \\
\hline \multirow{2}{*}{1} & Class Ia Type 1 \\
\hline & Class Ib Type 4 \\
\hline \multirow{2}{*}{2} & Class Ia Type 2 \\
\hline & Class Ib Type 2 \\
\hline \multirow{2}{*}{3} & Class Ia Type 3 \\
\hline & Class Ib Type 3 \\
\hline \multirow{2}{*}{4} & Class Ia Type 4 \\
\hline & Class Ib Type 1 \\
\hline \multirow{2}{*}{5} & Class Ia Type 5 \\
\hline & Class Ib Type 6 \\
\hline \multirow{2}{*}{6} & Class Ia Type 6 \\
\hline & Class Ib Type 5 \\
\hline \multirow{2}{*}{7} & Class Ia Type 7 \\
\hline & Class Ib Type 8 \\
\hline \multirow{2}{*}{8} & Class Ia Type 8 \\
\hline & Class Ib Type 7 \\
\hline \multirow{2}{*}{9} & Class Ia Type 9 \\
\hline & Class Ib Type 9 \\
\hline \multirow{2}{*}{10} & Class II Type 1 \\
\hline & Class II Type 4 \\
\hline 11 & Class II Type 2 \\
\hline 12 & Class II Type 3 \\
\hline \multirow{2}{*}{13} & Class II Type 5 \\
\hline & Class II Type 8 \\
\hline \multirow{2}{*}{14} & Class II Type 6 \\
\hline & Class II Type 7 \\
\hline 15 & Class II Type 9 \\
\hline
\end{tabular}

\section{5. $4 R$ Spherical LEMs}

Each 15 mechanisms in Table 5 was prototyped using creased joints in planar materials. By changing which link was the input, these 15 prototypes represent all 27 mechanisms shown in Table 3 . This is an effective way of 
quickly visualizing the motion, but has the limitation of possible interference between links. With LEMs, the range of joint motion and interference of links are often more limiting factors on motion than the mechanism type [15]. Knowing the mechanism type provides an understanding of possible linkage motion which will be advantageous in mechanism synthesis. From this starting point, the link shapes and joints can be designed appropriately. An example of a prototype is shown in Figure 4. The link lengths of this mechanism are $\alpha_{2}=45^{\circ}, \alpha_{3}=90^{\circ}, \alpha_{4}=45^{\circ}$, and $\alpha_{1}=90^{\circ}$ which makes this mechanism a Class Ia, Type 9 (see Table 3). This mechanism is capable of two planar positions.

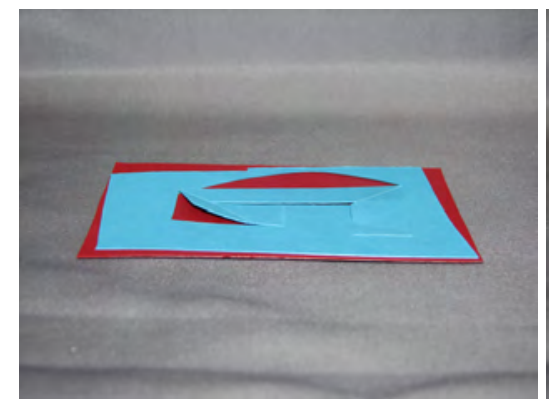

(a) In Planar Position

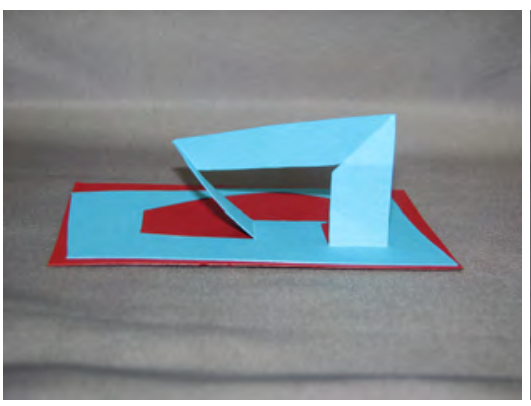

(b) In an Actuated Position

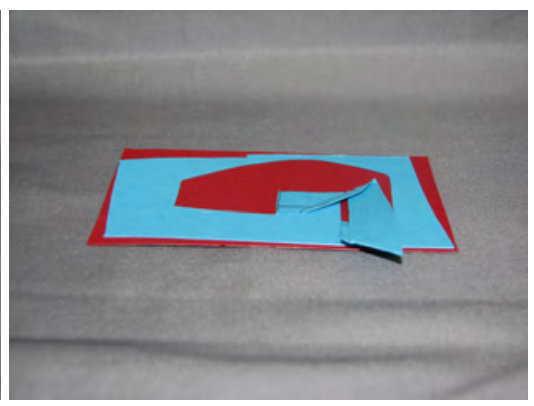

(c) In Second Planar Position

Figure 4: Spherical 4R LEM

\subsection{Six-Bar Spherical LEMs}

Spherical LEM equivalents of the Stephenson and Watt 6R chains are shown in Figures 5 and 6. The mechanism shown in these figures demonstrate the use arbitrary link shapes. However, even more so than with spherical $4 \mathrm{R}$ linkages, link collisions are common. The Stephenson chain is made of a spherical 4R and 5R chain, for which the analysis is more complicated than the Watt chain, which can be modeled as two spherical 4R linkages (see [17] and [18] for more information on the analysis of the Stephenson chain). The Stephenson 6R can be simplified by a partial shrinkage of the ternary link (not the ground link) [19]. This results in two serially connected 4R linkages that share the same sphere center. Examples of these types of mechanisms are shown in Section 3.7, Arrays of Spherical LEMs, of this work.

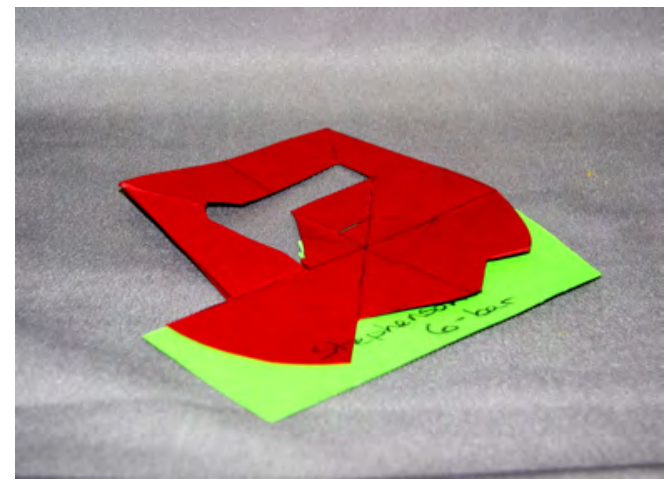

(a) In Planar Position

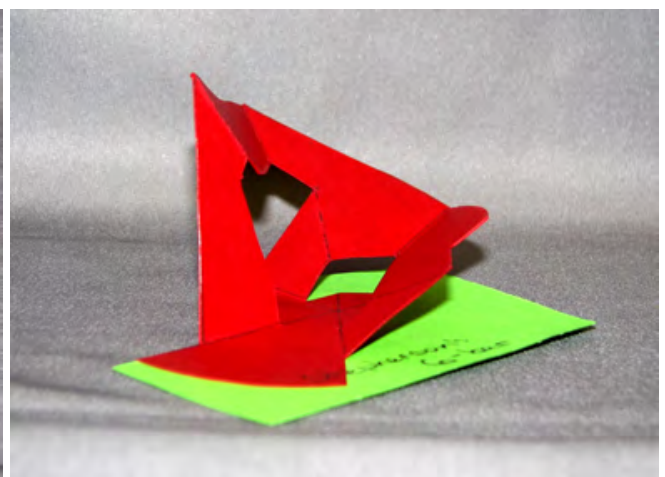

(b) In Actuated Position

Figure 5: Spherical 6R LEMs - Stephenson Chain 


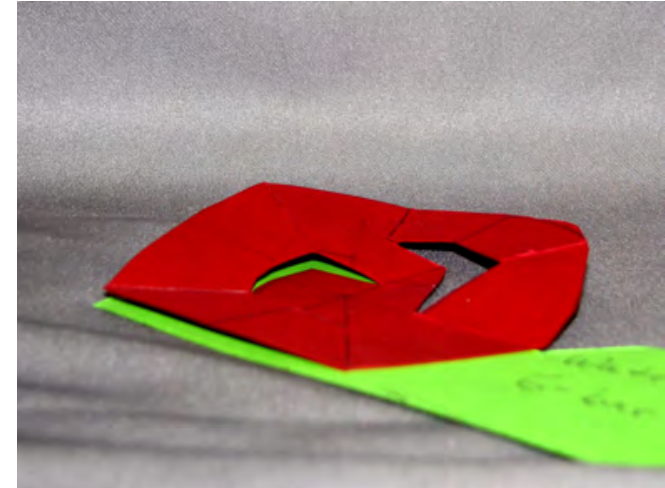

(a) In Planar Position

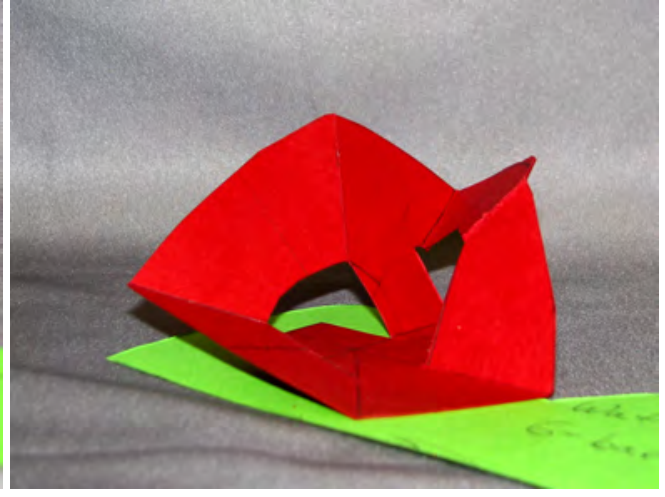

(b) In Actuated Position

Figure 6: Spherical 6R LEMs - Watt Chain

\subsection{Arrays of Spherical LEMs}

A LEM array is a patterned arrangement of LEMs [20]. This section derives patterned arrangements of spherical 4R linkages. Figure 7 is an array of two 4R LEMs. The serially connected spherical 4R linkage discussed by Makhsudyan et al. [21] is the basis of many of the following arrays. The output link of one 4R linkage is the input link to the other linkage. Notice the two 4R linkages do not share the same sphere center. However, the sphere centers do share a common axis. Continuing the same pattern of serially connected $4 \mathrm{R}$ chains, the array shown in Figure 8 contains four spherical $4 \mathrm{R}$ linkages, and four separate sphere centers. These arrays are built from identical 4R linkages. The resulting nine link mechanism is still a $1 \mathrm{DOF}$ linkage. Actuating one $4 \mathrm{R}$ linkage actuates the entire array. This pattern could be extended to as many spherical mechanisms as is necessary. Notice that in Figure 8 , the links of the spherical mechanism become disguised to demonstrate the ability to move desired shapes. The link lengths of each of the 4R linkages in the arrays shown in Figures 7 and 8 are $\alpha_{1}=\alpha_{3}=90^{\circ}, \alpha_{2}=\alpha_{4}=45^{\circ}$. With these link lengths, the $4 \mathrm{R}$ linkages are Class Ia, Type 9 mechanisms. Because Class Ia, Type 9 4R linkages are capable of two planar positions, the arrays are also capable of 2 planar positions.

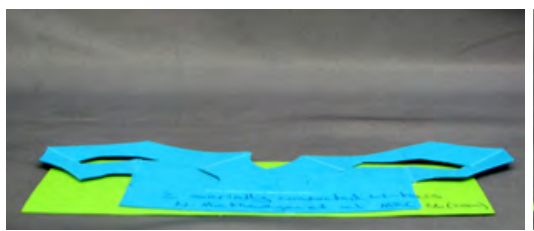

(a) In Planar Position

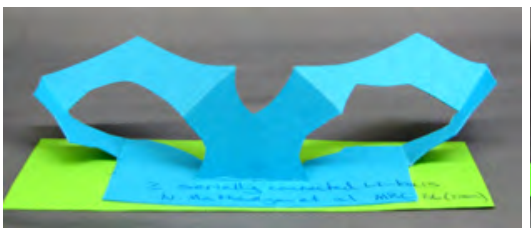

(b) In Actuated Position

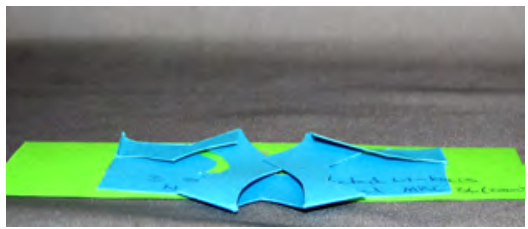

(c) In Second Planar Position

Figure 7: Two Serially Connected 4R LEMs

The array of spherical mechanisms shown in Figure 9 is similar to that shown in Figure 8 in that it is four serially connected 4R linkages, but the link lengths are different in this case. Taking the longer side of the box as the input link, the link lengths of each mechanism are $\alpha_{2}=90^{\circ}, \alpha_{3}=45^{\circ}, \alpha_{4}=135^{\circ}$, and $\alpha_{1}=90^{\circ}$. These linkages are Class Ib, Type 7 mechanisms (see Table 3). For this type of mechanism, the input link is limited by $\pm \theta_{\min }$, as defined by Equation (10). For this array, $\theta_{\min }=90^{\circ}$. In the planar state, $\theta=180^{\circ}$ and the input link can move to $\pm 90^{\circ}$. In its fully actuated position, $\theta=90^{\circ}$ for each $4 \mathrm{R}$ linkage in the array. Because the output links of each $4 \mathrm{R}$ linkage are the input links of another, those links are also limited to $\pm \theta_{\min }$. Notice that in contrast to the array in Figure 8, the "walls" (input and output links) of this array all reach $90^{\circ}$ at the same time and are limited in motion at that point. 


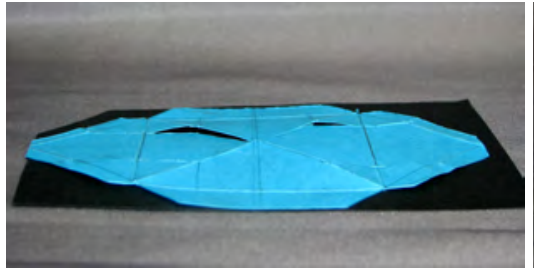

(a) In Planar Position

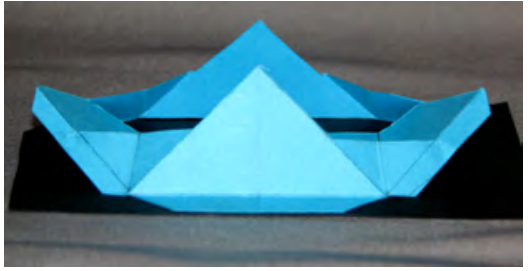

(b) In Actuated Position

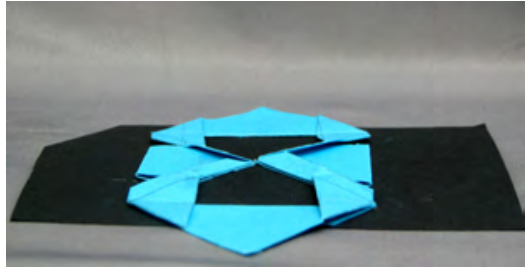

(c) In Second Planar Position

Figure 8: Four Serially Connected 4R LEMs

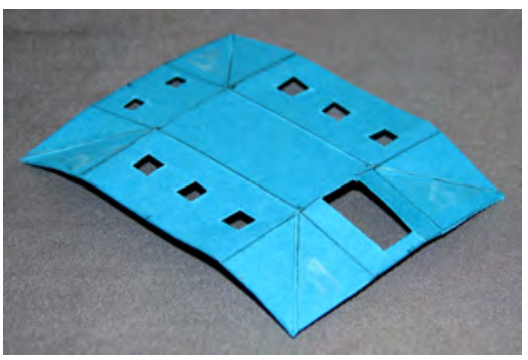

(a) In Planar Position

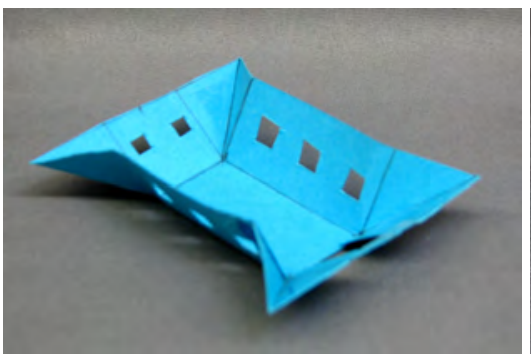

(b) Partially Actuated Position

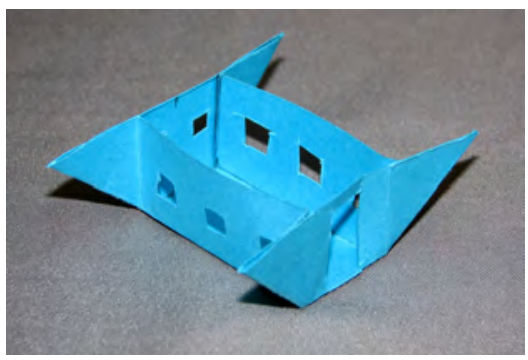

(c) In Actuated Position

Figure 9: Four Serially Connected 4R LEMs

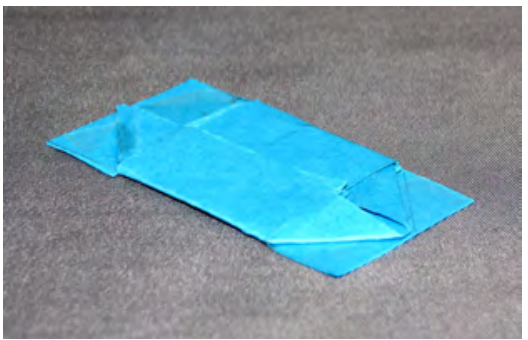

(a) In Planar Position

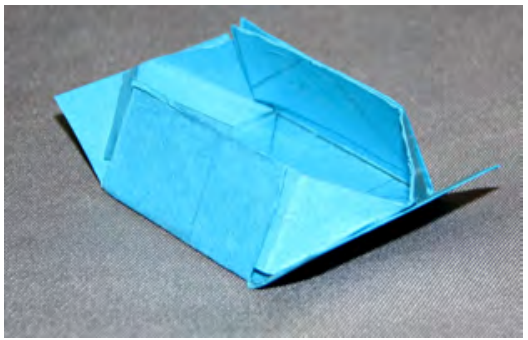

(b) Partially Actuated Position

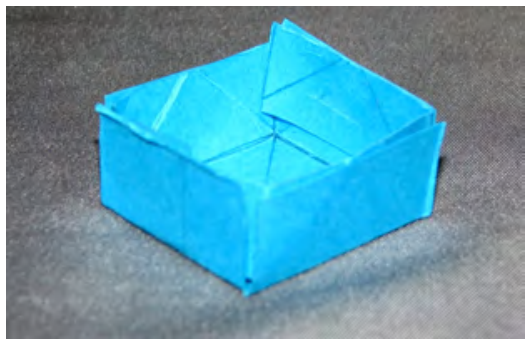

(c) In Actuated Position

Figure 10: Four Serially Connected 4R LEMs

The array in Figure 10 also features four serially connected spherical 4R linkages. Let the input links be the faces that fold towards the middle in the planar position. The link lengths for each spherical 4R linkage are $\alpha_{2}=90^{\circ}$, $\alpha_{3}=45^{\circ}, \alpha_{4}=45^{\circ}$, and $\alpha_{1}=90^{\circ}$. With these link lengths, the 4R linkages are Class Ia, Type 5 mechanisms. The input link is limited by $\pm \theta_{\max }$ as defined by Equation (9). For the $4 \mathrm{R}$ linkages in this array, $\theta_{\min }=90^{\circ}$, and $\theta=0^{\circ}$ in the planar position. In the fully actuated position $\theta=90^{\circ}$ for each $4 \mathrm{R}$ linkage. As with the previous example, the input and output links all reach $90^{\circ}$ at the same time and are limited in motion at that point. Although all three of the arrays discussed thus far have many similar characteristics, it has been shown that by decomposing the array into its spherical 4R linkages, the classification scheme developed by [5] and applied to spherical LEMs in this work can be used to predict the motion of the array as well as the limits of movement.

A different form of a spherical LEM array is a multi-layer LEM, or MLEM [22]. An example is shown in Figure 11. This mechanism is two serially connected $4 \mathrm{R}$ mechanisms on bottom, and two on top, sharing the same sphere center. It is interesting to note that even small inputs to the bottom mechanism quickly cause the motion of the top mechanism to reach its limit. The link lengths of the two mechanisms are identical, although the top mechanism 
is rotated $90^{\circ}$ relative to the bottom mechanism. The link lengths are the same for all $4 \mathrm{R}$ linkages in this array; $\alpha_{1}=\alpha_{3}=90^{\circ}$ and $\alpha_{2}=\alpha_{4}=45^{\circ}$, making each of the 4R linkages a Class Ia, Type 9 mechanism, capable of two planar positions. Connected as they are, the array is not capable of two planar positions due to interference between the top and bottom arrays.

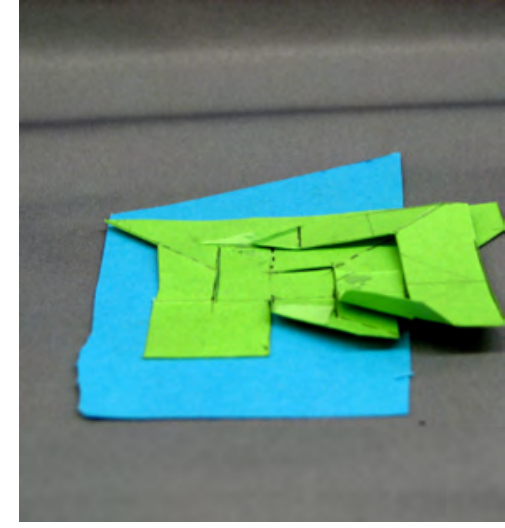

(a) In Planar Position

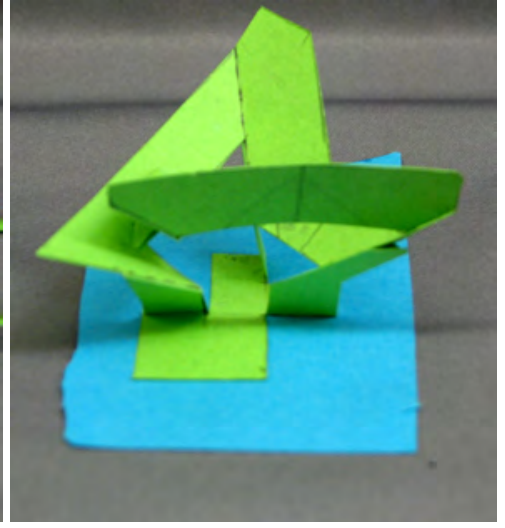

(b) In Actuated Position

Figure 11: Multi-layer LEM

Another form of a spherical LEM array is shown in Figure 12. This mechanism is four serially connected $4 \mathrm{R}$ linkages, all sharing the same sphere center. This array could also be modeled as two serially connected Stephenson 6R chains with partial shrinkage of their ternary links as previously discussed. The linkages "spiral" around the sphere center. This layout is similar to that used by Lusk et al. [23] on their Micro Helico-Kinematic Platform that involves an array of three spherical crank sliders that share the same sphere center. Although the mechanism shown in Figure 12 appears complex, it can be decomposed into simple 4R linkages. For each 4R linkage in this prototype, $\alpha_{1}=\alpha_{3}=90^{\circ}$ and $\alpha_{2}=\alpha_{4}=45^{\circ}$. Each $4 \mathrm{R}$ linkage is a Class Ia, Type 9 mechanism, capable of two planar positions.

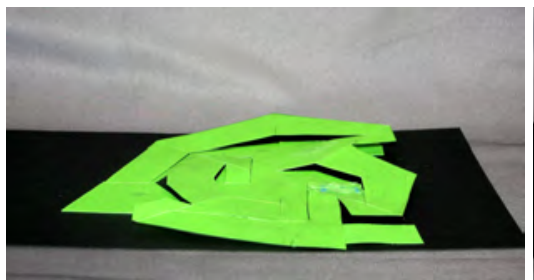

(a) In Planar Position

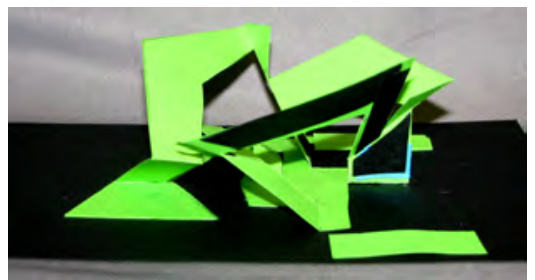

(b) In an Actuated Position

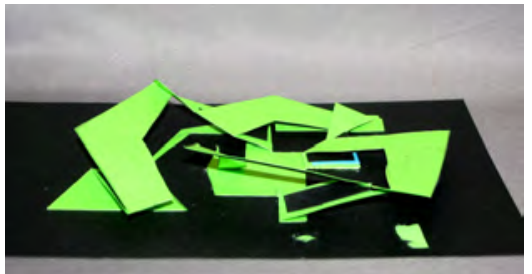

(c) In Second Planar Position

Figure 12: Four Serially Connected Spherical 4R LEMs Sharing the Same Sphere Center

\section{Conclusions}

A classification for spherical 4R LEMs was developed and the different types of possible spherical 4R LEMs were reported. Each of these mechanism types has unique motion characteristics which can be used as a starting point for mechanism synthesis. Examples of spherical 6R LEMs and E-quintets were demonstrated. Arrays of spherical 4R LEMs were prototyped and based on the types of spherical 4R linkages in the array, the motion of the array can be predicted. Other types of arrays were also demonstrated. These included MLEMs and multiple spherical 4R linkages that share the same sphere center. It has been shown that this classification can be extended to spherical mechanisms 
beyond the $4 R$ by decomposing the linkage into its respective $4 R$ chains. Based on the classification of the $4 R$ chains, the motion of the entire linkage can be predicted.

The extreme compactness of spherical LEMs makes them candidates for application with require complex motion using a small volume (e.g. space applications and deployable systems) and may be used as fundamental components in future surface morphing applications.

\section{Acknowledgments}

This material is based upon work supported by the National Science Foundation under Grant No. CMMI-0800606. Any opinions, findings, and conclusions or recommendations expressed in this material are those of the authors and do not necessarily reflect the views of the National Science Foundation.

\section{References}

[1] J. O. Jacobsen, B. G. Winder, L. L. Howell, S. P. Magleby, Lamina emergent mechanisms and their basic elements, Journal of Mechanisms and Robotics 2 (2010) 1-9.

[2] L. L. Howell, Compliant Mechanisms, Wiley-Interscience, 2001.

[3] C. H. Chiang, Kinematics of Spherical Mechanisms, Cambridge University Press, 1988.

[4] P. Larochelle, J. Dooley, A. P. Murray, J. M. McCarthy, SPHINX - Software for synthesizing spherical mechanisms, in: NSF Design and Manufacturing Systems Conference, Charlotte, North Carolina. Proceedings of the 1993 NSF Design and Manufacturing Systems Conference.

[5] J. J. Cervantes-Sánchez, H. I. Medellín-Castillo, A robust classification scheme for spherical 4R linkages, Mechanism and Machine Theory 37 (2002) 1145-1163.

[6] C. R. Barker, A complete classification of planar four-bar linkages, Mechanism and Machine Theory 20 (1985) $535-554$.

[7] A. Svoboda, Computing Mechanisms and Linkages, MIT Radiation Laboratory Series. No. 27., McGraw-Hill, 1948.

[8] D. P. Martin, A. P. Murray, Developing classifications for synthesizing, refining, and animating planar mechanisms, ASME Conference Proceedings 2002 (2002) 1397-1403.

[9] J. M. McCarthy, Geometric Design of Linkages, Springer, 1 edition, 2000.

[10] M. Savage, J. Hall, Unique descriptions of all spherical Four-Bar linkages, Journal of Engineering for Industry 92 (1970) 559-563.

[11] H. Su, C. L. Collins, J. M. McCarthy, Classification of RRSS linkages, Mechanism and Machine Theory 37 (2002) $1413-1433$.

[12] H. I. Medellín-Castillo, J. J. Cervantes-Sánchez, An improved mobility analysis for spherical 4R linkages, Mechanism and Machine Theory 40 (2005) 931-947.

[13] C. P. Lusk, L. L. Howell, Components, building blocks, and demonstrations of spherical mechanisms in microelectromechanical systems, Journal of Mechanical Design, Transactions of the ASME 130 (2008).

[14] B. G. Winder, S. P. Magleby, L. L. Howell, Kinematic representations of pop-up paper mechanisms, Journal of Mechanisms and Robotics 1 (2009) $1-10$

[15] B. G. Winder, S. P. Magleby, L. L. Howell, A study of joints suitable for lamina emergent mechanisms, in: 2008 ASME International Design Engineering Technical Conferences and Computers and Information in Engineering Conference, DETC 2008, August 3, 2008 - August 6, 2008, volume 2 of 2008 Proceedings of the ASME International Design Engineering Technical Conferences and Computers and Information in Engineering Conference, DETC 2008, ASME, New York City, NY, United states, 2009, pp. 339-349.

[16] J. O. Jacobsen, G. Chen, L. L. Howell, S. P. Magleby, Lamina emergent torsional (LET) joint, Mechanism and Machine Theory 44 (2009) 2098-2109.

[17] S. Hernandez, S. Bai, J. Angeles, The design of a chain of spherical stephenson mechanisms for a gearless robotic Pitch-Roll wrist, Journal of Mechanical Design 128 (2006) 422-429.

[18] C. W. Wampler, Displacement analysis of spherical mechanisms having three or fewer loops, Journal of Mechanical Design 126 (2004) 93-100.

[19] R. Norton, Design of Machinery, McGraw-Hill Science/Engineering/Math, 4 edition, 2007.

[20] N. B. Albrechtsen, S. P. Magleby, L. L. Howell, Identifying potential applications for lamina emergent mechansism using techonology push product development, in: 34th Mechanisms and Robotics Conference, presented at - 2010 ASME International Design Engineering Technical Conferences and Computers and Information in Engineering Conference, IDETC/CIE2010, August 15, 2010 - August 18, 2010.

[21] N. Makhsudyan, R. Djavakhyan, V. Arakelian, Comparative analysis and synthesis of six-bar mechanisms formed by two serially connected spherical and planar four-bar linkages, Mechanics Research Communications 36 (2009) 162-168.

[22] P. S. Gollnick, S. P. Magleby, L. L. Howell, An introduction to multi-layer lamina emergent mechanisms (2011). In review for publication in Journal of Mechanical Design.

[23] C. P. Lusk, L. L. Howell, A micro Helico-Kinematic platform via spherical crank sliders, ASME Conference Proceedings 2004 (2004) $131-141$ 